\title{
TYPE + CODE VII: Typographic selfie + code
}

\author{
Yeohyun Ahn \\ University of Wisconsin Madison \\ 4200 University Ave \#103 \\ Madison, WI, 53705, USA \\ yahn27@wisc.edu
}

\begin{abstract}
A selfie is a self-portrait digital photograph that one has taken of oneself, typically taken with a smartphone or web camera and shared by social media. The typographic selfie + CODE is an extension of Selfie + CODE, which is a collection of generative selfies taken by the author to raise awareness of Asian female faculty being isolated and marginal in a predominantly white institution of the United States. The computational processes expend the concepts of traditional self-portraits to generative visual languages conveying specific thoughts or feelings. Typography is a form of art to make written language expressive by using letter forms and text. Based on the type choices, different emotions and moods can be visually expressed through the generative selfies. The typographic selfie + CODE is visual research with diverse typefaces to embed visual expression into generative selfies.
\end{abstract}

Self portrait. Generative art. Typography. Visual expression. Selfie. Generative photography.

\section{INTRODUCTION}

A selfie is a photograph typically one taken with a smartphone or webcam and shared by social media. Over 1 million selfies are now taken every day. Selfies are not always as spontaneous as they seem. A selfie is a form of art. It can be a communication tool purposely.

The typographic selfie + CODE is an extension of Selfie + CODE, a collection of generative selfies taken by the author. The computational processes expand the concepts of traditional self-portraits to computational visual languages conveying specific thoughts or feelings. The author started taking her generative selfies in 2015 to raise awareness of Asian female faculty being isolated in a predominantly white institution. It is extended to a medium, typography, with the title, Typographic Selfie + CODE.

Her generative selfies capture psychological moments to express those individual identities being devalued by a homogeneous institution in the United States and are shared by social media. Her virtual supporting system, 'Like', at Facebook, enabled her to persist, and survive in a regionally isolated and exclusive community. Eventually, it has brought her psychological reconciliation and healing to succeed in dealing with difficulties. Each typeface has a visual personality. The typographic selfie + CODE is visual research with diverse typefaces to embed visual expression and emotion into generative selfies.

\section{BACKGROUND}

Asian female faculty is a small but growing population of higher education in the United States. Asian female faculty in the United States may struggle to navigate between two exclusive communities, Asian, and American. Professors in Asian cultures were predominantly conceived as male positions. It was a socially privileged and highly respected position in the patriarchal Asian cultures. Asian female faculty occasionally encounters circumstances to be less inclusive and invisibly exclusive in the Asian communities since their emerging populations may threaten their wellestablished patriarchal hierarchy being controlled and led by men in the Asian communities. The Asian communities predominately pressure Asian women to perform traditional roles as mothers and housewives.

Asian female faculty who are bonded with this culture are inevitably destined to be isolated and marginalised within their ethnic groups, and to encounter criticisms toward their non-traditional roles. Furthermore, the Asian female faculty suffers from stereotypes of being perceived as 
uncomplaining, perseverant and submissive to authority in predominately white institutions. Their classroom may be a minefield of student resistance and negative attitudes (Nguyen 2016). According to the paper, 'Women of Color Faculty at the University of Michigan: Recruitment, Retention, and Campus Climate', women faculty of colour might be the most marginalised faculty on US campuses. Challenging areas were isolation, high attrition, student evaluations, peer perception, more service responsibilities, and others (Cox 2008).

\section{SELFIE + CODE}

Selfie + CODE is a collection of the generative selfies taken by the author since 2015 . It has been capturing psychological moments, to express how those individual identities are devalued and deconstructed by homogeneous institutions and ethnocentric groups in the United States (Figure 1).

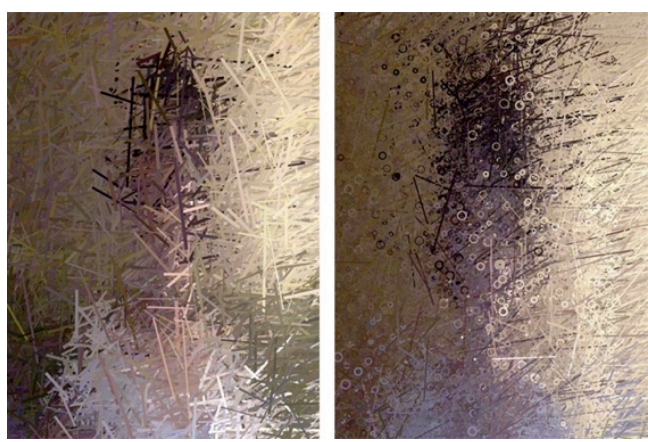

Figure 1: Selfie + CODE.

The visual style was inspired by Impressionism, which is a 19th-century art movement that captures a moment, such as Claude Monet's Sunrise; and Expressionism, which expresses inner troubles and feelings of anxiety rather than technical skills or beauty, which are otherwise traditional goals of art (Figure 2).

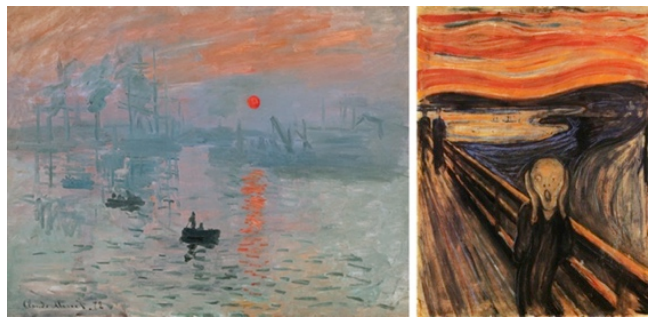

Figure 2. Left: Sunrise (1872), Claude Monet, Right: The Scream (1893), Edvard Munch.

Selfie + CODE was initially implemented by using Mirror library, developed by Daniel Shiffman in Processing. It transforms each pixel from a realtime video source to a rectangle, based on the levels of brightness by using an internal web camera. Each shape is transformed into a line, to draw the moments being brushed off and added as several variables, functions, and colour palettes to express the visual theme (Figure 3).
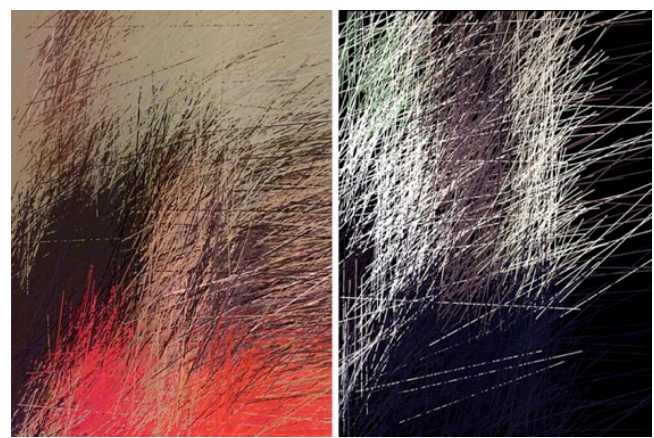

Figure 3. Initial sketches by the Being Ignored 1.0.

The series of selfies were taken in the author's office space in a regionally isolated white institution, to represent a space for Asian female faculty on US campuses. The office light was intentionally controlled to be darker to express the atmosphere. An internal web camera captured the author's self-portrait photograph by using a computer algorithm, Being Ignored 1.0, and eliminated the facial expression to express being treated as less valued, unremarkable, and not worthy of attention. It was taken using different angles and levels of the light repeatedly and sequentially. The process was similar to professional photos that are taken in a photo studio (Figure 4).
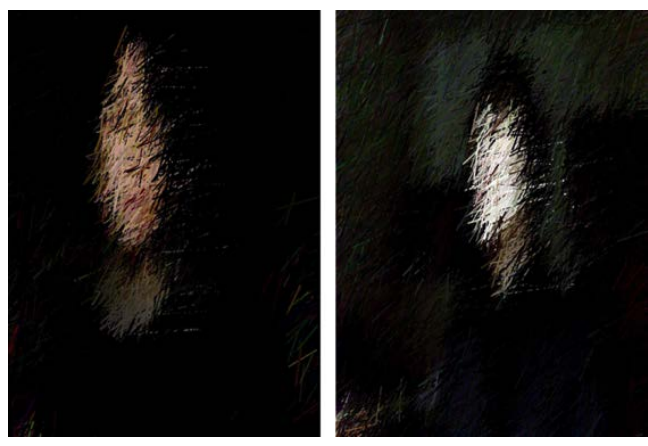

Figure 4. Final Outputs.

\section{TYPOGRAPHIC SELFIE + CODE}

Typography is an art form where text is expressive and highly visual. The selfie is a self-expression to convey a mood or share a story through social media. Commonly, facial expression is used to convey a wide range of emotions through a selfie. Each typeface has a personality reflecting specific visual atmospheres and moods. The visual research uses typeface on selfie as facial expression to convey feelings and thoughts. The following typefaces are chosen to embed into selfie directly to investigate how selfies integrate with typefaces to be expressive and visual. 


\subsection{Helvetica}

Helvetica is a modern, intelligent and stylish typeface designed by Max Miedinger in 1957. It is among the most widely used sans-serif typefaces (Hyndman 2016; Figure 5).
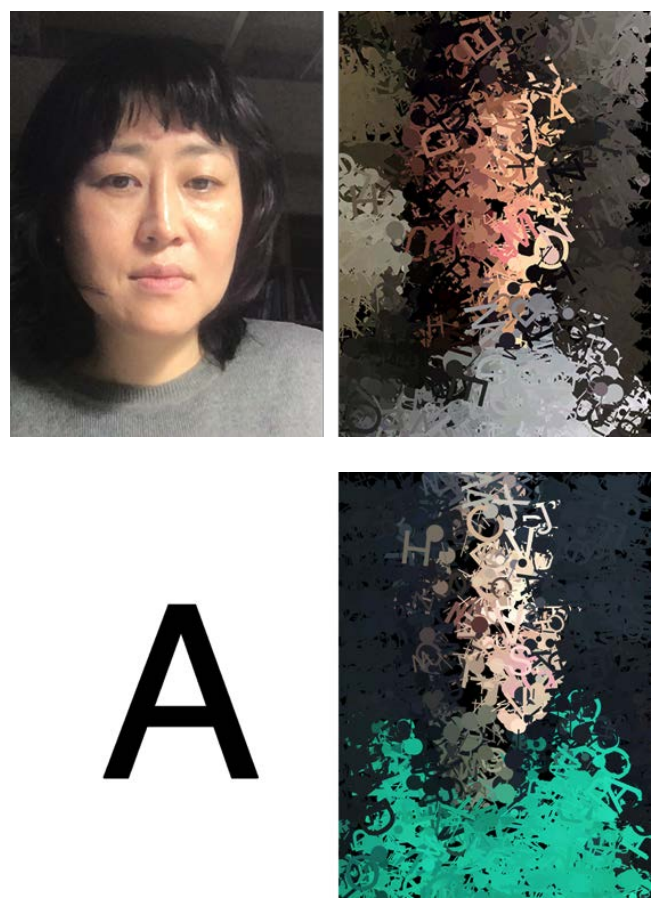

Figure 5: Top left: Original Selfie. Top right: Generative Selfie with Helvetica. Bottom left: Helvetica A. Bottom right: Generative Selfie with Helvetica.

\subsection{Times}

Times is an intellectual, confident, academic and professional typeface designed by Stanley Morison Victor Lardent in 1931. It was commissioned by the British newspaper, The Times. It is one of the most popular and influential typefaces in history and a stared typefaces on desktop computers (Hyndman 2016; Figure 6).
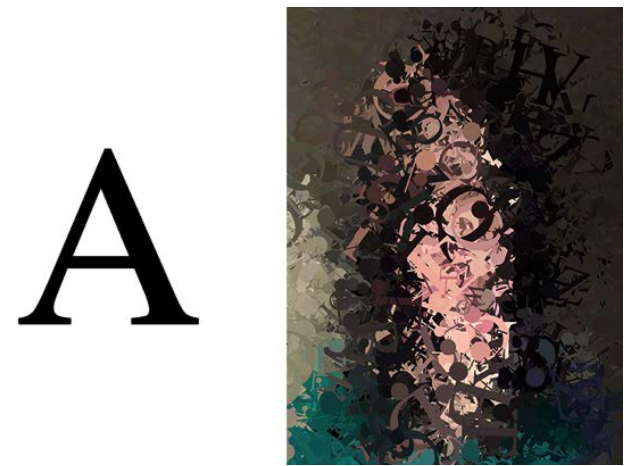

Figure 6 Left: Times A. Right: Generative Selfie with Times.

\subsection{Futura}

Futura is a modern, practical, comfortable, and capable sans serif typeface designed by Paul Renner in 1927 (Hyndman 2016; Figure 7).
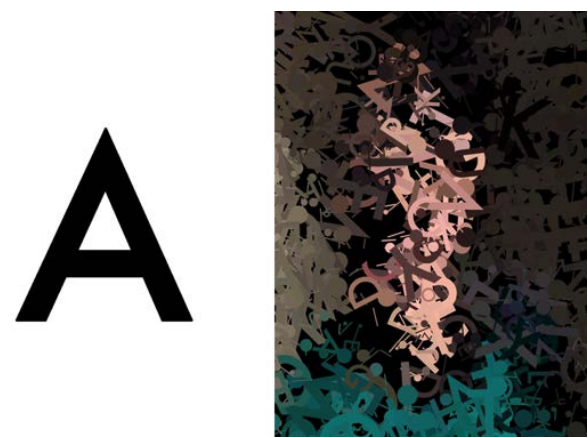

Figure 7: Left: Futura A. Right: Generative Selfie with Futura.

\subsection{Didot}

Didot is a sophisticated, polished and professional typeface developed in the period 1784-1811 by Didot family (Hyndman 2016; Figure 8).
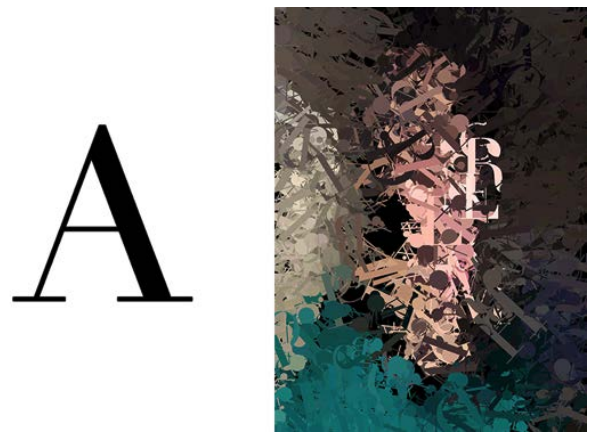

Figure 8: Left: Didot A, Right: Generative Selfie with Didot.

\subsection{Baskerville}

Baskerville is a traditional, credible, and neutral typeface designed by John Baskerville in 1750s. It is still popular in book design. (Hyndman 2016; Figure 9).
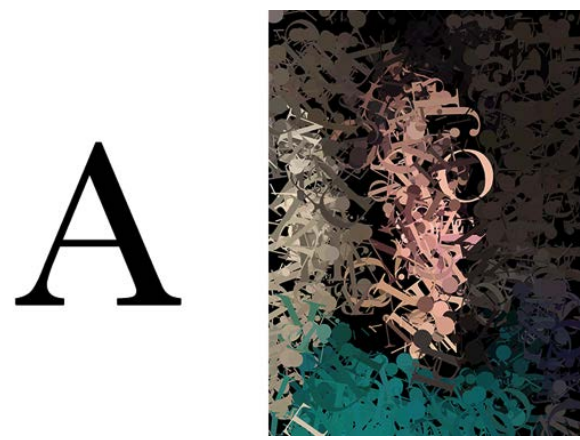

Figure 9: Left: Baskerville A. Right: Generative Selfie with Baskerville. 


\subsection{Asmelina Harley}

Asmelina Harley is an elegant calligraphy script typeface designed by Arif Dwi at Kotak Kuning Studio in 2019 (Figure 10).
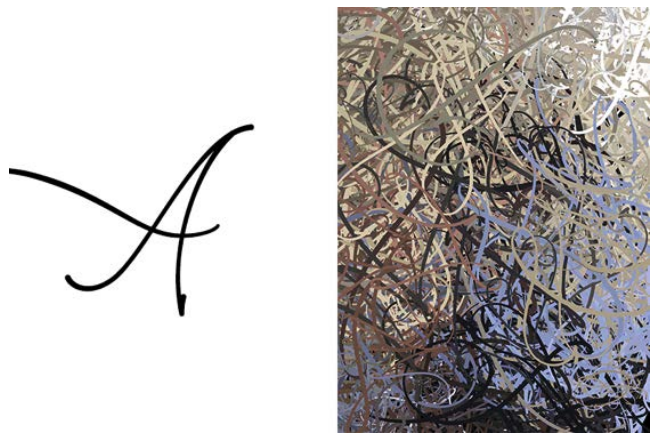

Figure 10: Left: Asmelina Harley A. Right: Generative Selfie with Asmelina Harley.

\section{CONCLUSION}

The author started taking the generative selfies in 2015 to bring awareness of Asian female faculty who are detached, isolated and alienated in a predominately white institution of the United States. They have been shared through her social media. The virtual supporting system, 'Like', at Facebook made her endure her life-time challenges in an isolated and exclusive community of the United States. It brought her vision to initiate a multidisciplinary art and design project, Social Homelessness on US Campuses, http://www.socialhomelessness.com, which is a supporting system for Asian female faculty in predominately white institutions of the United States.

In 2019, the collection of the generative selfies entitled Selfie + CODE was realised as a solo exhibition format. Each typeface has an individual, distinctive character. For instance, Helvetica is modern and stylish typeface. Times are intellectual and academic typeface. The Typographic Selfie + CODE is visual research project intended to show how to use typeface in generative selfies to convey feeling and thoughts, as an extended typographic practice and application. The research demonstrates how traditional typographic principles and practices, including typeface choice and arrangement, as well as visual expression, may be applicable for generative selfies. It shows the possibilities to use the personality of each typeface in expressive and visually appealing generative art.

The future development of this research will involve more diverse typefaces, aligning with the history and styles of these. Also, it will be more collaborative, with visual elements such as lines, circle and curves providing visual eloquence, which is both meaningful and indicative (Figure 11).

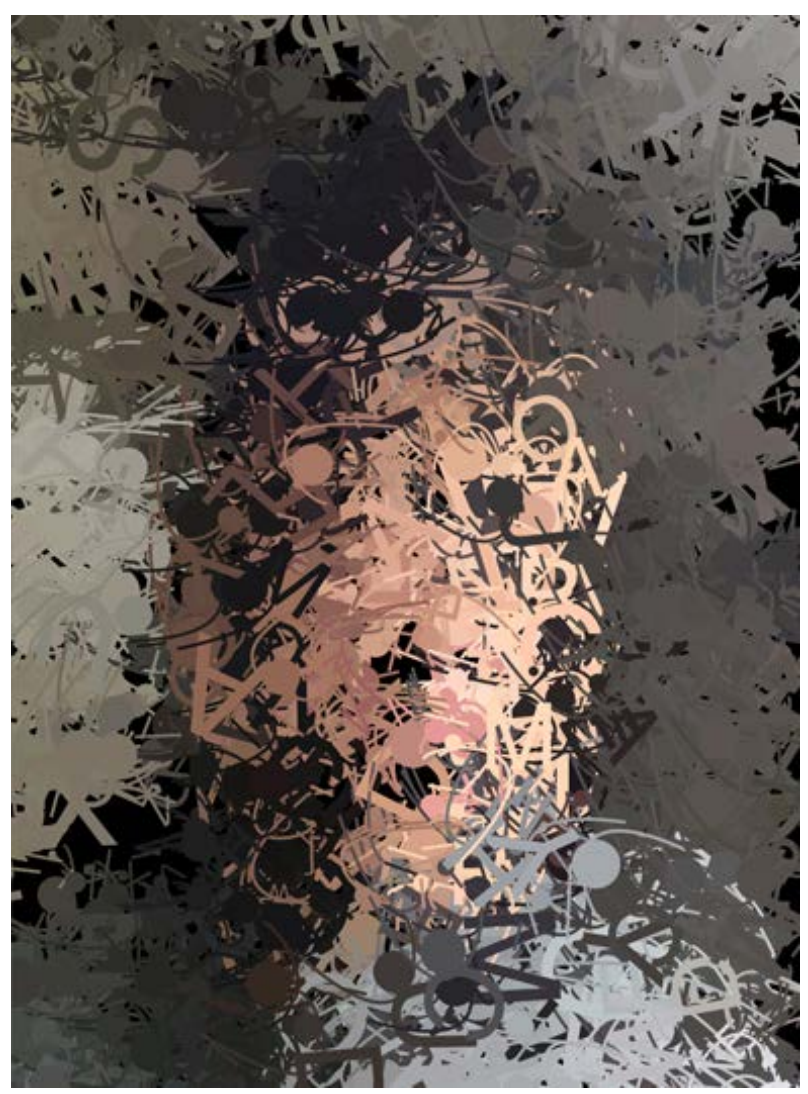

Figure 11: Generative Selfie with Helvetica, Circles, and Curves.

\section{REFERENCES}

Cox, A. (2008) Women of Color Faculty at the University of Michigan: Recruitment, Retention, and Campus Climate. University of Michigan, MI, p.2.

Hyndman, S. (2016) Why Fonts Matter. Gingko Press, CA, pp.80-81.

Nguyen, C. F. (2016) Asian American Women Faculty: Stereotypes and Triumphs. University of San Francisco, San Francisco, CA, pp.129-136. 